\title{
Report on the Study of Radiation Damage in Calcium Fluoride and Magnesium Fluoride Crystals for use in Excimer Laser Applications
}

Research performed for CRADA No. BNL-C-96-10 between

\author{
Optovac \\ North Brookfield, MA \\ and \\ Brookhaven National Laboratory \\ Upton, NY \\ October 4, 1999 \\ RECEIVED \\ JAN 0321300 \\ OSTI
}

\begin{abstract}
A study was performed to investigate the effects of radiation damage in calcium fluoride and magnesium fluoride crystals caused by gamma rays and UV photons from excimer lasers. The purpose was to study and correlate the damage caused by these two different mechanisms in various types of material used for fabricating optical elements in high power excimer lasers and lens systems of lithography tools. These optical systems are easily damaged by the laser itself, and it is necessary to use only the most radiation resistant materials for certain key êlements. It was found that a clear correlation exists between the radiation induced damage caused by high energy gamma rays and that produced by UV photons from the excimer laser. This correlation allows a simple procedure to be developed to select the most radiation resistant material at the ingot level, which would be later used to fabricate various components of the optical system. This avoids incurring the additional cost of fabricating actual optical elements with material that would later be damaged under prolonged use. The result of this screening procedure can result in a considerable savings in the overall cost of the lens and laser system.
\end{abstract}




\section{DISCLAIMER}

This report was prepared as an account of work sponsored by an agency of the United States Government. Neither the United States Government nor any agency thereof, nor any of their employees, make any warranty, express or implied, or assumes any legal liability or responsibility for the accuracy, completeness, or usefulness of any information, apparatus, product, or process disclosed, or represents that its use would not infringe privately owned rights. Reference herein to any specific commercial product, process, or service by trade name, trademark, manufacturer, or otherwise does not necessarily constitute or imply its endorsement, recommendation, or favoring by the United States Government or any agency thereof. The views and opinions of authors expressed herein do not necessarily state or reflect those of the United States Government or any agency thereof. 


\section{DISCLAIMER}

Portions of this document may be illegible in electronic image products. Images are produced from the best available original document. 


\section{INTRODUCTION}

Excimer lasers were first commercialized in the late 1970's. For many years they were an interesting new scientific tool for academic research, but by the mid 1980's, new industrial and medical applications began to be investigated. These applications included using excimer lasers for ablating the lens in the eye to correct for myopia, hyperopia and astigmatism. In parallel, new excimer lasers began to be developed as a replacement for mercury lamps in optical lithography tools for the manufacture of micro processors and DRAMs. Ultraviolet photons from an excimer laser were also seen to have industrial applications in micromachining.

In order to achieve ever smaller feature sizes in semi-conductor chips, engineers designing lithography tools used in the manufacturing process have been forced to go to shorter and shorter wavelengths to achieve their desired resolution. In 1975, the wavelength used was the g-line of a mercury lamp at a wavelength of $436 \mathrm{~nm}$, which achieved a resolution of 1.4 microns. In 1987, the wavelength was reduced to $365 \mathrm{~nm}$ [the I-line a mercury lamp], and the resolution improved to 0.7 microns. The need to further improve the resolution forced the industry to change the light source from a mercury lamp to a $\mathrm{KrF}$ excimer laser emitting light at $248 \mathrm{~nm}$. This led to improved resolution, first to 0.25 microns, and then to 0.18 microns. For the present state of the art, a further reduction in wavelength to $193 \mathrm{~nm}$ using a ArF excimer laser enables a resolution of $\sim 100 \mathrm{~nm}$ to be achieved. This wavelength is also the wavelength of choice for opthamalic applications, although the fluences used there are considerably higher than those used in lithography. In the future, smaller feature sizes will require even shorter wavelengths, such as the $157 \mathrm{~nm}$ light produced with a $F_{2}$ excimer laser.

By 1995 , the semi-conductor industry had adopted the use of excimer lasers as a light source in lithographic tools, and in 1996 the FDA first approved the use of ArF excimers for the treatment of myopia. Although these applications differ widely, they share common optical materials, namely, calcium fluoride and magnesium fluoride components for chamber windows, beamsplitters and output couplers. The applications also share a need to have a low rate of failure. One major cause of downtime was caused by having to replace optical components because of induced absorption caused by the laser radiation interacting with low levels of impurities in the optics. 
The primary goal of this CRADA was to establish whether there was a correlation between the absorption induced by excimer lasers and that induced by gamma radiation. If such a correlation could be established, then irradiating material in its early stages of fabrication with gamma radiation would offer a much more efficient means of pre-testing the material before it went through the very costly manufacturing procedure to fabricate it into high quality, coated laser optics.

\section{PROCEDURE}

The first initial tests of optical materials was done using a $193 \mathrm{~nm}$ excimer laser at MIT Lincoln Lab and the Gamma Ray Irradiation Facility (GRIF) at Brookhaven National Lab. Samples were exposed to $1-2 \times 10^{6}$ laser pulses, and the laser induced damage was measured in terms of the change in optical transmission as a function of wavelength in the range of 200-800 nm. For the laser exposure, the damage was typically confined to a small region of the sample, such that most of the sample was unaffected. The same samples were then exposed to gamma ray irradiation, which exposed the entire sample, and the optical transmission measured over the same wavelength range, both in the previously laser damaged region, as well as the undamaged region.

A clear correlation was found between the laser induced damage and the gamma ray induced damage. Figure 1 summarizes this correlation for a set of $\mathrm{CaF}_{2}$ samples, and Figure 2 shows the same correlation for a set of $\mathrm{MgF}_{2}$ samples. Both the laser induced and gamma ray induced damage produced color centers in the crystal which resulted in the formation of absorption bands, as shown in Figure 3 . In $\mathrm{CaF}_{2}$, the band at $395 \mathrm{~nm}$, as well as others produced in the 400-600 $\mathrm{nm}$ range; are observed for gamma ray doses as low as a few tens of kilorads. In $\mathrm{MgF}_{2}$, typically a single absorption peak is produced at $256 \mathrm{~nm}$, but the threshold for damage is typically much higher $(\sim 1 \mathrm{Mrad})$.

While the predominant absorption caused by both laser induced and gamma ray induced damage occurs in the visible wavelength range, the greatest concern for damage in the optical system is in the far UV and vacuum ultraviolet. In order to study this region, a vacuum ultraviolet spectrometer was constructed at $\mathrm{BNL}$ which was used to extend the optical absorption measurements from $200 \mathrm{~nm}$ down to $125 \mathrm{~nm}$. This covered the 
region of the $193 \mathrm{~nm}$ ArF laser, as well as allowing for future study at 157 $\mathrm{nm}$ with the $\mathrm{F}_{2}$ laser. The spectrometer was based on a MacPhearson Model 234/302 VUV monochrometer which included a deuterium light source, a computer controlled monochromator, and a special sample compartment designed to hold the crystal samples.

The VUV system was used in conjunction with the Gamma Ray Irradiation Facility at BNL to study the damage produced in materials immediately after gamma ray irradiation. Due to the close proximity of the VUV spectrometer to GRIF, it was possible to measure samples typically within less than 15 minutes after irradiation. This minimized any effects of recovery in the samples, and provided much more accurate information on the actual amount of damage produced. Figure 4 shows some typical results from the VUV scan of several $\mathrm{CaF}_{2}$ samples in the range from $150-300 \mathrm{~nm}$. There are no significant absorption bands produced in the regions of interest for laser applications, which was a general finding from this study. In particular, the region around $193 \mathrm{~nm}$ is free of any absorption bands, but there was a general increase in the overall level of absorption in this region for some samples.

In order to study the actual laser damage, a laser induced absorption facility was developed at Optovac. This system was based on a Lambda Physik LPX-240 ArF excimer laser and was used to measure changes in the optical absorption of samples at $193 \mathrm{~nm}$ due to exposure to laser light. With this facility, it is possible to carry out systematic studies of the laser damage in numerous samples produced under different conditions, and to measure the induced absorption coefficient with relatively high precision. Figure 5 shows the results of one such study, which shows the change in transmission at 193 $\mathrm{nm}$ as a function of the number of laser pulses for samples produced with different surface treatment processes. This demonstrates one important finding from this study, in that surface damage contributes significantly to the overall laser induced damage in many samples. It is expected that surface absorption will be an even more significant effect at $157 \mathrm{~nm}$, and therefore a more detailed investigation into the causes and elimination of surface absorbing layers would be of great benefit. 
SUMMARY

Significant Accomplishments:

- A VUV spectrometer system was developed at BNL which permitted. extending the measurements of optical absorption below $200 \mathrm{~nm}$ which could be used in conjunction with the BNL Gamma Ray Irradiation Facility. Results from samples which were tested showed no significant absorption bands in the region of interest for laser applications.

- An excimer laser irradiation system was developed at Optovac which allowed measuring the change in optical absorption due to $193 \mathrm{~nm}$ laser light. It was found that this damage consisted of contributions from surface effects as well as from bulk absorption. Other factors, such as purity of raw material and growth conditions, also affected the radiation hardness of the materials tested.

- A clear correlation was established between the laser induced damage and gamma ray induced damage in both $\mathrm{CaF}_{2}$ and $\mathrm{MgF}_{2}$ materials. $\mathrm{A}$ screening procedure using gamma ray irradiation can therefore be used to identify suitable material for further processing into laser optics.

-Significant Problems:

- The demands placed on the materials used for lithography are extremely high and require not only inherently robust materials, but also stringent testing procedures to accurately determine the level of absorption produced by actual laser light. Unfortunately, there is no accepted standard protocol in the industry for measuring initial and induced absorption. However, testing materials to an individual customer's specification often requires making very high precision measurements of the absorption coefficient $\left(\sim .0005 \mathrm{~cm}^{-1}\right)$ in relatively short samples. As present, this is beyond the capability of the BNL VUV system, and is even extremely difficult with any commercially available spectrometer.

- Achieving credibility for a gamma ray irradiation test will require a large number of samples to be tested and the results to be correlated with measurements made at other laboratories. However, since the damage of interest in laser optics is that caused by the laser itself with normal operating fluences $\left(\sim 1 \mathrm{~mJ} / \mathrm{cm}^{2}\right)$ and rates $(\sim 200-300 \mathrm{~Hz})$, it can require up to several months to obtain a single laser exposure under these 
conditions. This limits the ability to study the laser damage in the number of samples required, and to precisely correlate this damage with that produced by gamma rays.

Industry Realized Benefits:

- Demonstrating that a correlation exists between gamma ray induced absorption and laser induced absorption, and that both mechanisms lead to the same type of color center formation, gives confidence that gamma ray irradiation may be a cost effective way of screening material. A simple, cost effective screening procedure can in principle be developed which will enable rejection of poor quality material even at the ingot stage. However, more detailed work is required to develop such a procedure that would be acceptable to the eventual customers these materials.

- The establishment of a laser induced absorption facility at Optovac has enabled statistically significant numbers of samples to be tested. Having this facility at Optovac has also greatly enhanced the ability to provide immediate feedback to the production process from the results of these tests.

\section{Laboratory Realized Benefits:}

- A high quality VUV specirometer system was built and operated at BNL which is now available to extend the present work, as well as for the study of other materials and other applications.

- BNL has now developed the expertise for carrying out gamma ray irradiation studies and measuring laser optic materials in the UV and VUV. This expertise can be applied to a variety of other applications of interest to the laboratory. 


\section{Recommended Follow-on Work:}

- Carry out further systematic studies with larger numbers of samples grown from the most recent pure raw material in order to establish the correlation between laser damage and gamma ray damage at $193 \mathrm{~nm}$ at the level of precision required by industry.

- Develop the actual gamma ray screening procedure. This would involve developing a procedure with higher throughput than the current system would allow, but could be easily extended upon from the present apparatus.

- Improve the resolution of the BNL VUV spectrometer by incorporating a dual-beam measurement system. This would significantly improve the precision of the data obtained in the VUV region and would permit measurements of surface damage in the materials being studied.

- Extend the present work to include a more detailed study of the radiation damage effects from both the laser and gamma rays at $157 \mathrm{~nm}$. This would be of considerable interest for the next generation of lithography instruments.

Potential Benefits From Follow-on Work:

- Optovac could make use of the Gamma Ray Irradiation Facility at BNL to carry out the screening procedure of its laser optics grade material.

- Significant improvements could be made in the quality of laser optics by improving both the bulk material and the surface treatment of the finished optics. This would require further studies of these effects with higher precision absorption measurements in the VUV for both laser and gamma ray induced damage.

- Improvements in the understanding of the damage mechanism in fluoride laser optics would be of great benefit to both $193 \mathrm{~nm}$ and future $157 \mathrm{~nm}$ lithography applications.

- A higher precision VUV spectrometer would be of benefit for other studies of materials that are of interest to the research program at BNL. 
Figure 1. $\mathrm{CaF}_{2}$ 2M laser pulses

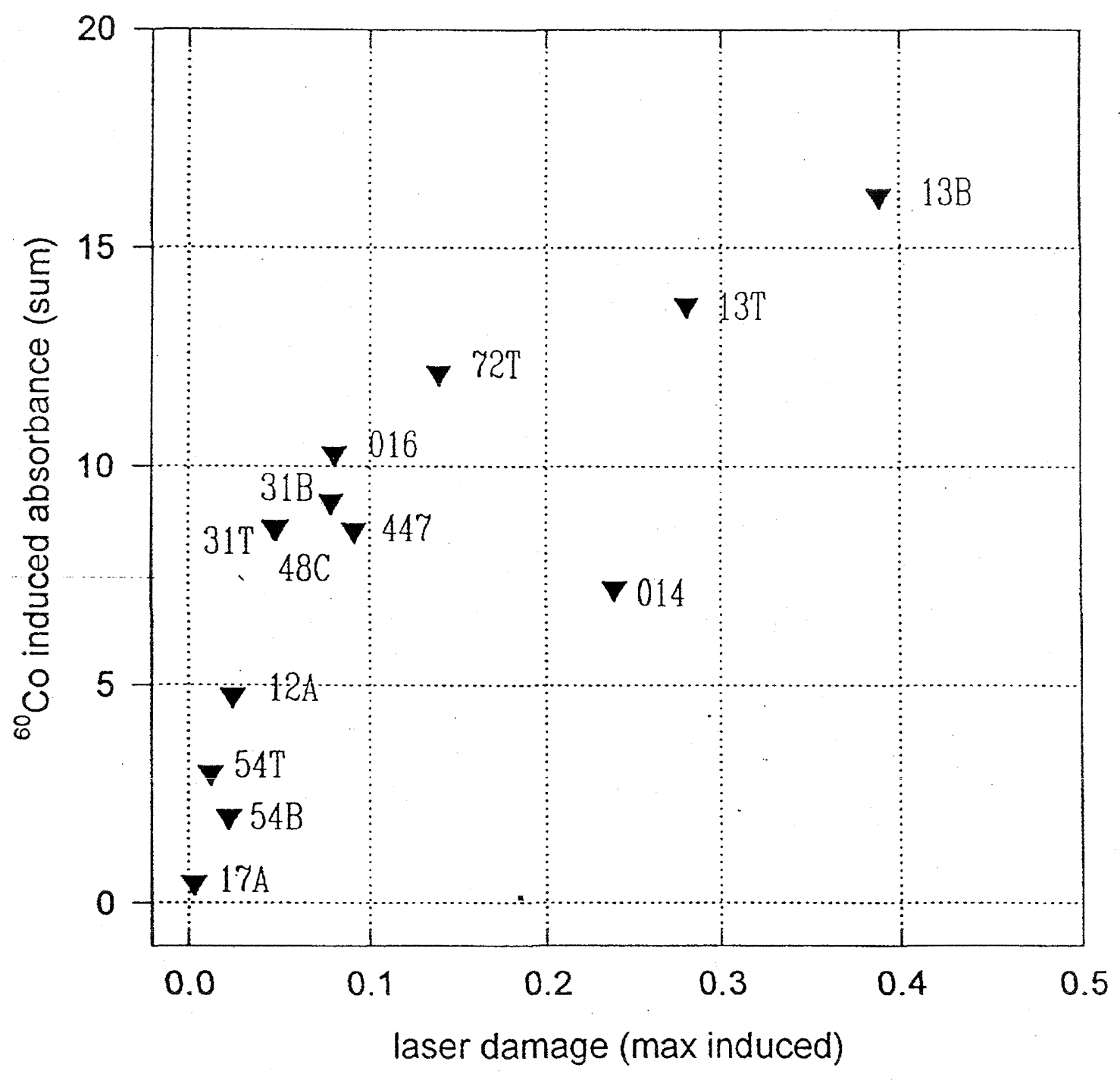


Figure 2. $\mathrm{MgF}_{2}$ 2M laser pulses previously undamaged region

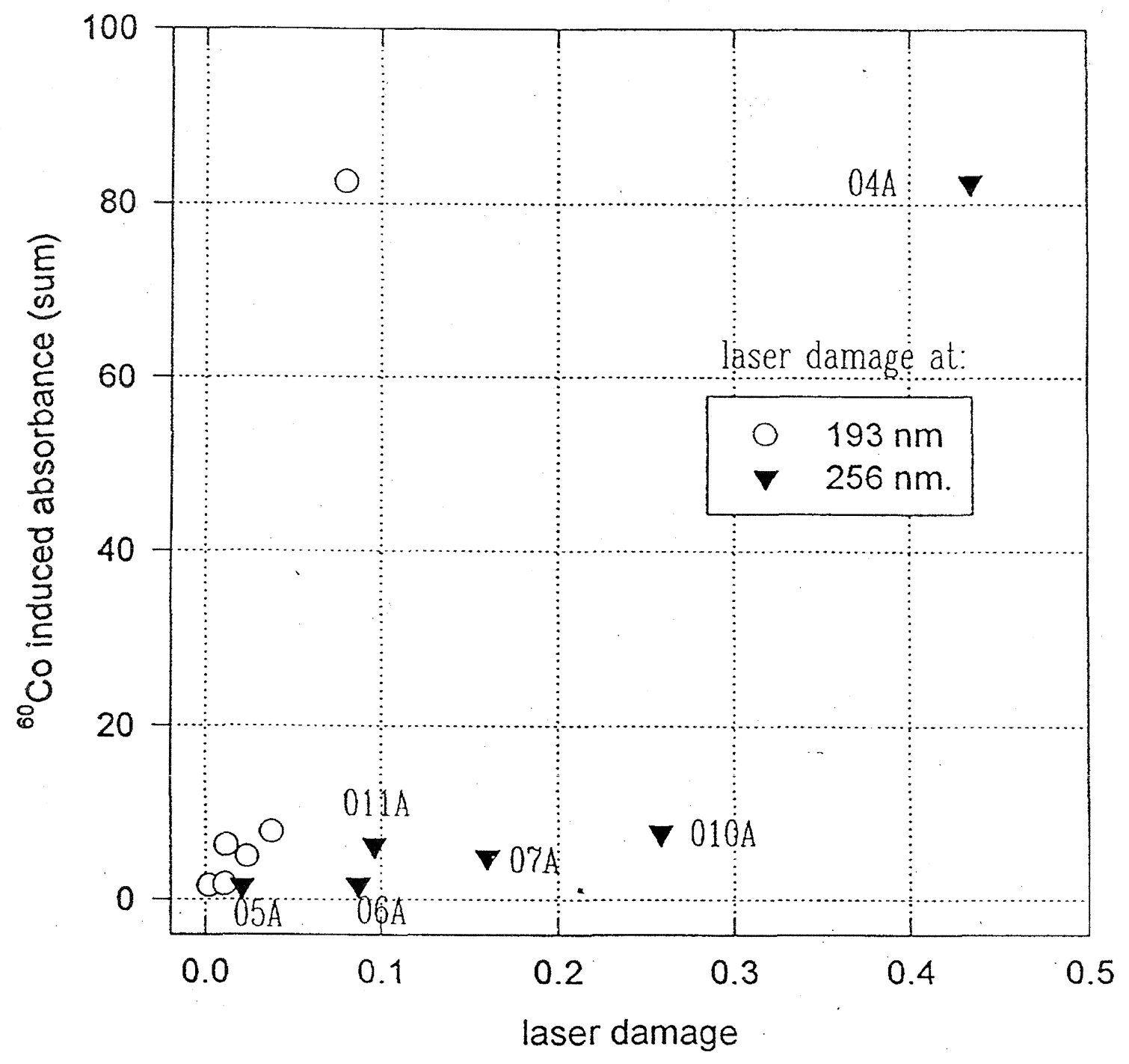


Figure 3

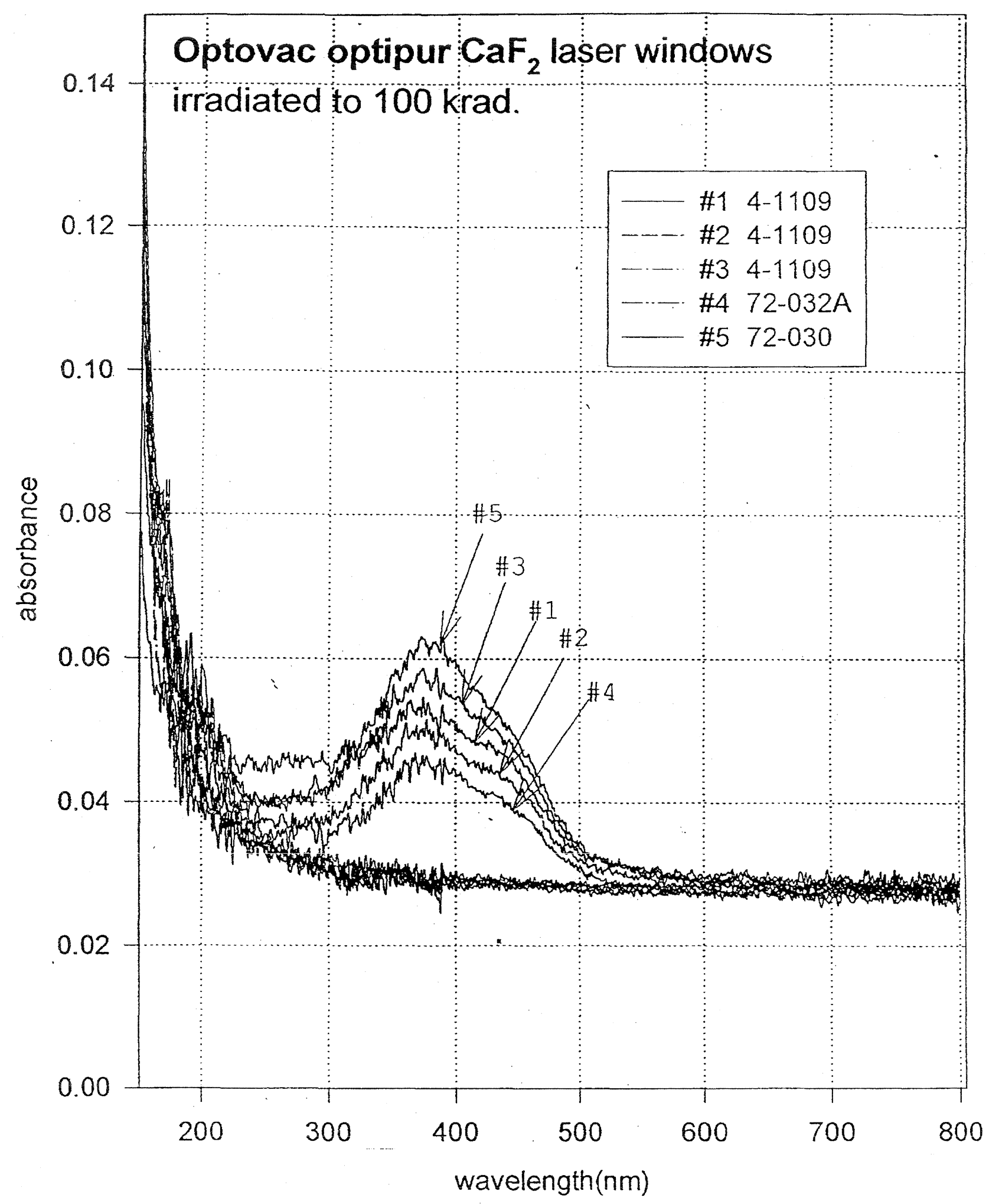


Figure 4

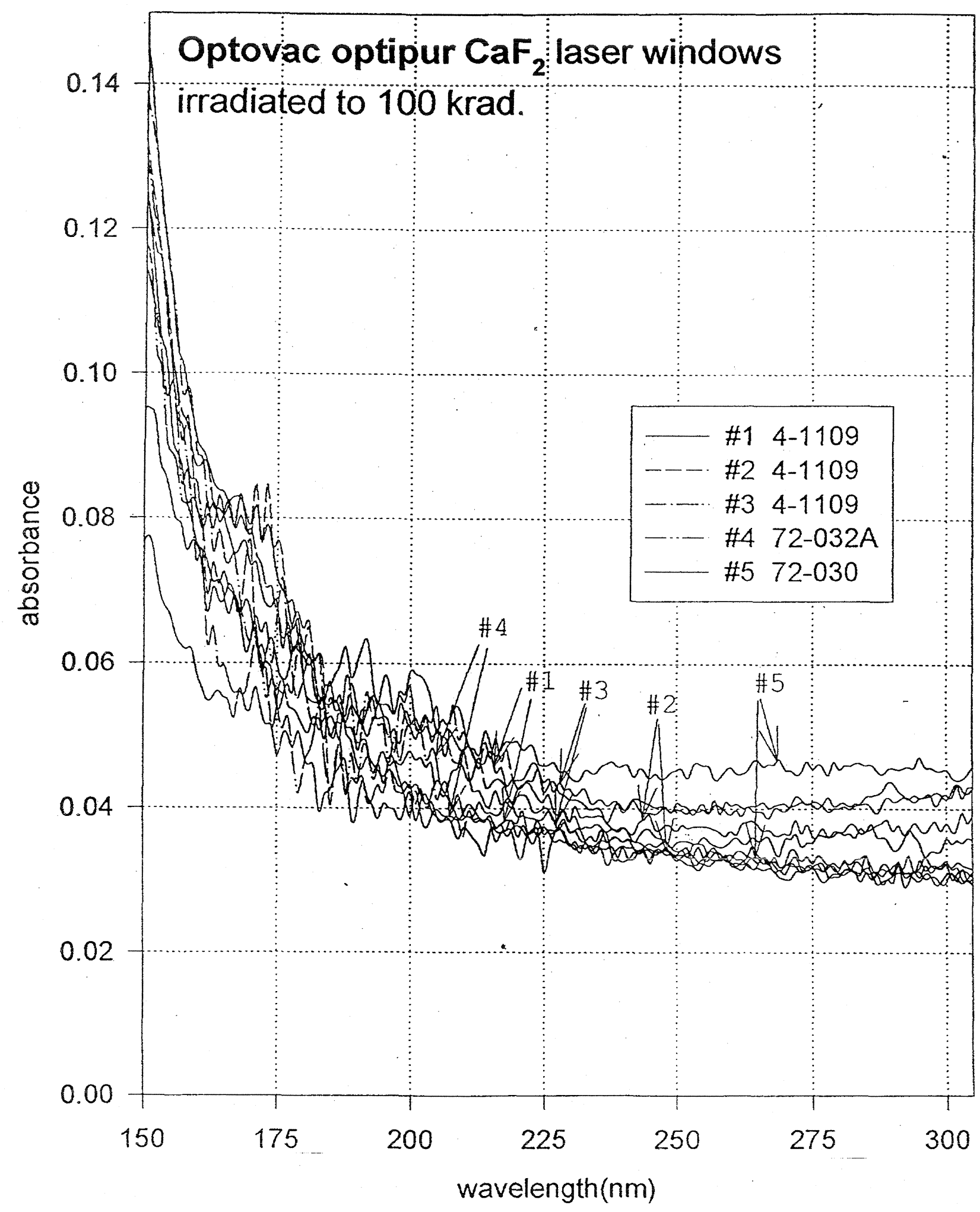




\section{Figure 5}

\section{$193 \mathrm{~nm}$ Laser Induced Transmission Change in CaF2 Windows $v$. Surface Process}

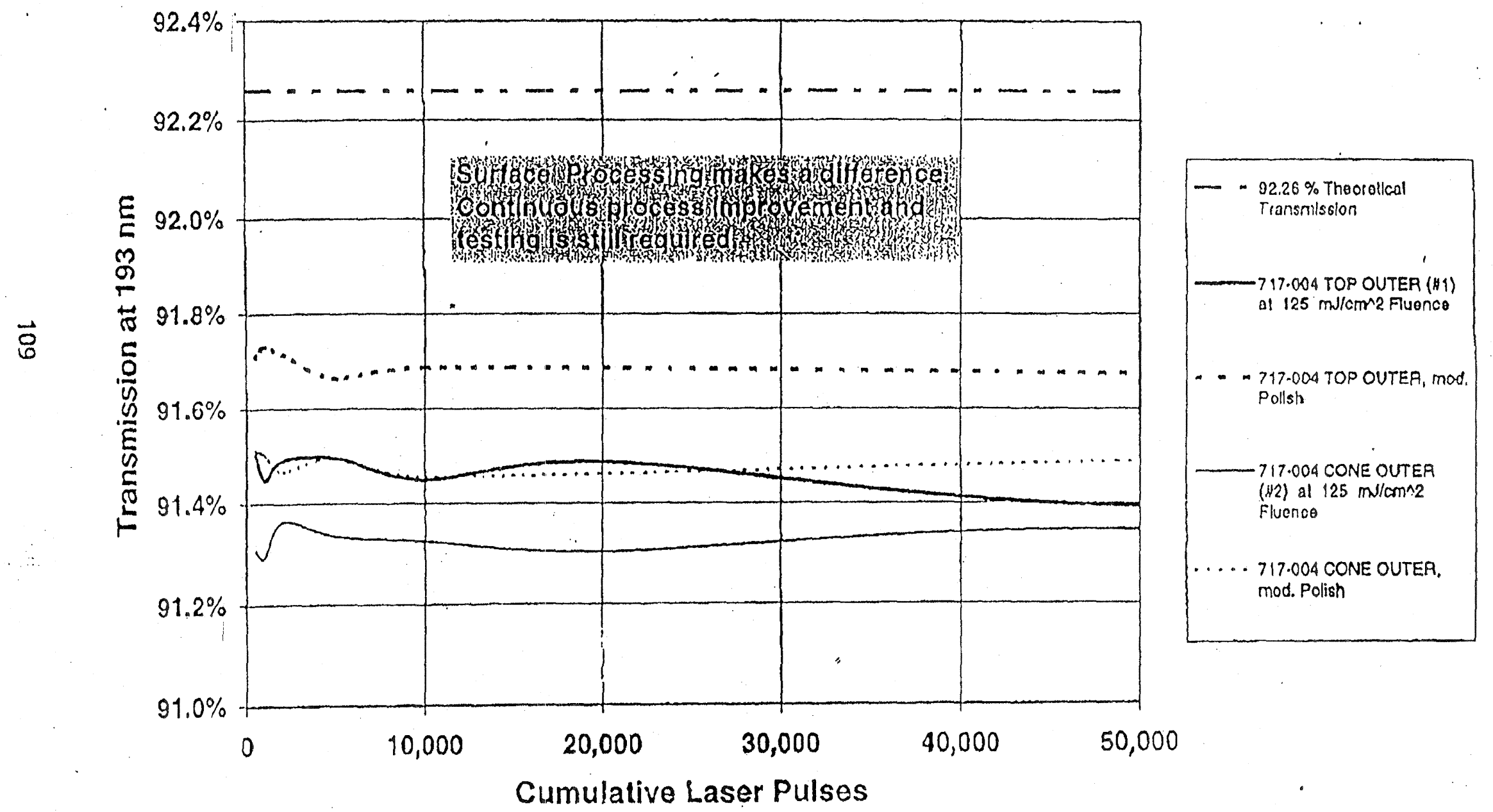

